\title{
Intelligent Robotics for Smart Agriculture
}

\author{
Bin $\mathrm{He}^{*}$ \\ Shanghai Key Laboratory of Intelligent Manufacturing and Robotics, Shanghai University, China
}

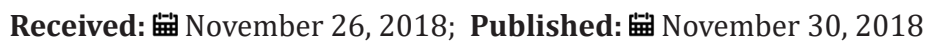

*Corresponding author: Bin He, Shanghai Key Laboratory of Intelligent Manufacturing and Robotics, Shanghai University, China

\begin{abstract}
Smart agriculture is a cyber-physical agriculture management concept involved in the observing, measuring and responding variability of crops in the agriculture management cycle. This paper is devoted to the intelligent robotics for smart agriculture. After the robotics demand analysis, the key technologies are proposed step by step, including robotics vision modeling and decision making, robotics pattern recognition and human-computer interaction decision, robotics function development and test verification.

Keywords: Agricultural Robotics; Tomato Picking Robotics; Smart Agriculture; Smart Farm; Intelligent Robotics; Underactuated Robotics
\end{abstract}

\section{Introduction}

In recent years, advanced technology in industry has driven agricultural development. Various countries have invested a lot of funds to research intelligent agricultural robotics, which have greatly promoted agricultural development. As the agriculture is the foundation of economic development, agriculture development plays an important role in China's economic development. In recent years, with the reduction of the farmers and the increase in labor costs, the demand has further increased for agricultural production. Modern agricultural robots with high efficiency, high intelligence and low production cost will change the traditional agricultural mode and have become an indispensable productive datum for contemporary agricultural production. Robotics in agriculture could greatly improve the productive efficiency so that further improve agricultural economic benefits. The combination of highly intelligent robots and agriculture creates a new world for new urban modern agricultural production [1].

It has begun earlier that the research of agricultural robots with some results. Fruit and vegetable picking robots, transplanting robots and grafting robots have begun to enter the application stage. The Dutch van Henten et al. developed a 7-DoFs (degrees of freedom) cucumber picking robot that uses multiple spectra to identify cucumbers, whose recognition rate could be up to $80 \%$ and the picking cycle was about 45 second [1-10]. Japan Hayashi et al. developed a 3-DoFs strawberry picking robot that used machine vision to determine ripeness of strawberry and positioning ripe fruit with about 55\% success rate. The modern agricultural intelligent robot is the foundation to complete the task of smart agricultural production, with integrating sensing technology, detection technology, artificial intelligence technology, communication technology, image recognition technology, precision and system integration technology, etc. It shows great innovation and industrialization prospects in improving agricultural productivity, changing agricultural production patterns, and realizing the scale, diversification and precision of agriculture and so on $[2,3]$.

\section{Key Technology of Agricultural Robotics}

Facility agriculture is a typical labor-intensive industry, and the harvest of fresh fruits and vegetables is the key that takes up the most labor and is the most difficult to achieve mechanize work. At home and abroad, the picking process relies on a large number of labors to complete, and the automation of picking operations has become a realistic demand for the development of facility agriculture production. In order to improve the level of fruit harvesting automation, a new intelligent tomato picking robotics system for smart agriculture is developing. The tomato picking robotics system realizes autonomous navigation by machine vision, recognizes and spatially locates tomatoes through machine vision, and manipulates the end effector by the articulates underactuated mechanical arm for carry out the picking process of tomatoes to achieve intelligent picking by adsorb tomatoes, clamp and cut tomatoes handles [4-6]. 


\section{Design Solution}

Fruits and vegetables have the characteristics of complex growth environment, easy damage and inconsistent maturity, and the fruit and vegetable picking are labor-intensive work. The harvesting is much more complicated than grain harvesting. Fruit and vegetable picking have high labor intensity, long time and high harvesting cost; therefore, it is extremely urgent to develop intelligent equipment for picking fruit and vegetable. At the same time, it is also a necessary with high efficiency, high yield and intelligence of facility agriculture. The tomato picking jobs determines environmental conditions and objectives of space picking, and optimizes structural parameter, analyzes kinematics, simulates dynamics and correct structure and plausibility of the model for the picking manipulator. A flexible picking robot is proposed to solve the problem, as the traditional robot end effector is easy to cause tomato fruit picking damage. The way that consider using new materials to manufacture flexible grippers, apply hydraulic, pneumatic, electric or mechanical transmission to drive the claws and adopt new flexible picking could reduce the number of sensors on the robot arm and the difficulty of the coordinated control of the operating system. It could be more compact through the optimization of the robotics mechanism. With the improvement of the flexibility and agility for the robot arm and the end effector, it could avoid obstacle without injury picking, and improve the success rate, and then reduce the rate of damage fruit [7].

Solving the multi-sensor data fusion problem of greenhouse tomato harvesting robot, developing the multi-source information acquisition hardware and software of picking decisions, researching robotic joint space trajectory planning and trajectory tracking control algorithm. Through analysis and comparison of the advantages and disadvantages of each algorithm and combine the picking robot system structural characteristics and the requirement of fruit picking operations to propose the motion planning method for efficient and non-destructive picking and adaptive control strategy of robots. It intends to develop a set of open control system to increase the versatility of the picking robot. The control system picks different fruits and vegetables by change the structural parameters of end-effector and mechanical arm. Thereby can improve the utilization of the system and reduce agricultural production costs. Developing a physical prototype of fruit and vegetable picking robots such as tomatoes, and establishing an agricultural robot application demonstration base to promote the development of fruit and vegetable picking robot technology [8-10].

\section{Conclusion}

With the acceleration of urban-rural integration, the shortage of agricultural labor and rising labor costs has seriously affected the development of the fruit and vegetable agriculture. The picking robotics is necessary in the efficient, high yield and intelligent development of smart facility agriculture. The project intends to conduct in-depth research on the key technologies of tomato picking robots to develop physical prototypes of tomatoes picking robots and to establish application demonstration bases of agriculture robot to promote the development of fruit and vegetable picking robotics.

\section{Acknowledgement}

The work was supported by Shanghai Science and Technology Commission Project (Grant No. 18391900900).

\section{References}

1. He B (2018) Artificial Intelligent for Intelligent Manufacturing and Robotics. Robotics and Automation Engineering Journal 1(5): 555-575.

2. He B, Xue HJ, Liu LL, etc. Rigid-flexible coupling virtual prototypingbased approach to the failure mode, effects, and criticality analysis. International Journal of Advanced Manufacturing Technology, p. 1-23.

3. He B (2018) Intelligent manufacturing and robotics in offshore engineering. Aeronautics and Aerospace Open Access Journal 2(2): 9596.

4. He B (2018) Underactuated robotics in aerospace and agricultural engineering. Aeronautics and Aerospace Open Access Journal 2(2): 8586.

5. Zaman MTU, Ahmed MS, Sabbir H, Shakhawat H, Jamal GRA (2015) Design and Construction of a Multipurpose Robot. International Journal of Automation. Control and Intelligent Systems 1(2): 34-46.

6. Alexander D (2015) Adaptive Locomotion Control System for Modular Robots. International Journal of Automation, Control and Intelligent Systems 1(4): 92-96.

7. Mozafari Ehsan, Abolghasemi V, Ferdowsi Saideh (2016) Providing a Method for Tile Troubleshooting Using Thin Display and Image Processing. International Journal of Automation, Control and Intelligent Systems 2(1): 1-8.

8. He B, Zhang P, Liu W (2017) Dynamics analysis and numerical simulation of a novel underactuated robot wrist. Proceedings of the Institution of Mechanical Engineers, Part B: Journal of Engineering Manufacture 231(12): 2145-2158

9. He B, Liu W, Huang S (2015) Kinematics analysis and numerical simulation of a novel underactuated robot wrist. Proceedings of the Institution of Mechanical Engineers, Part B: Journal of Engineering Manufacture 229(8): 1429-1448.

10. He B, Hou S, Deng, Z, Jintao Cao, Wenzhen Liu (2014) Workspace analysis of a novel underactuated robot wrist based on virtual prototyping. International Journal of Advanced Manufacturing Technology 72(1-4): 531-541. 
(c) (P) This work is licensed under Creative

To Submit Your Article Click Here: Submit Article

DOI: 10.32474/ARME.2018.01.000117

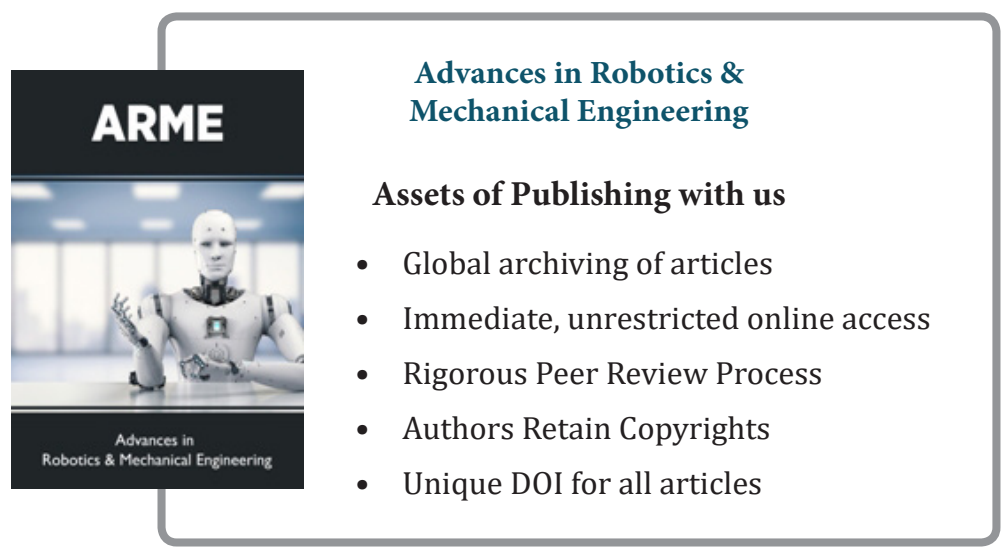

